\title{
METODOLOGIA PARA DETECÇÃO E TRATAMENTO DE FALHAS EM SISTEMAS DE MANUFATURA ATRAVÉS DE REDE DE PETRI
}

Tese apresentada à Escola Politécnica da

Universidade de São Paulo para obtenção do título de Doutor em Engenharia.

São Paulo

2002 


\section{METODOLOGIA PARA DETECÇÃO E TRATAMENTO DE FALHAS EM SISTEMAS DE MANUFATURA ATRAVÉS DE REDE DE PETRI}

Tese apresentada à Escola Politécnica da Universidade de São Paulo para obtenção do título de Doutor em Engenharia.

Área de concentração:

Engenharia Mecânica, opção MECATRÔNICA.

Orientador:

Prof. Dr. Paulo Eigi Miyagi.

São Paulo

2002 
Ficha CATALOGRÁFICA ELABORADA PELA BIBLIOTECA DE ENGENHARIA MeCÂNiCA/NAVAl Da EsCOLA POLITÉCNICA EPMN -USP

Martinez Riascos, Luis Alberto

Metodologia para Detecção e Tratamento de Falhas em Sistemas de Manufatura através de Redes de Petri, São Paulo, 2002 $161 \mathrm{p}$.

Tese (Doutorado) - Escola Politécnica da Universidade de São Paulo. Departamento de Engenharia Mecatrônica e Sistemas Mecânicos.

1. Falhas em Sistemas de Manufatura 2. Redes de Petri 3. Abordagem Proposta (Redes de Petri Distribuídas) 4. Estudos de caso 5. Simulador de Redes Distribuídas I. Universidade de São Paulo. Escola Politécnica. Departamento de Engenharia Mecatrônica e Sistemas Mecânicos II. t 
A minha mãe e aos meus irmãos Carmen Helena

e Carlos Arturo, que sempre acreditaram em mim. 


\section{AGRADECIMENTOS}

Inicialmente quero agradecer ao Brasil e aos brasileiros por me acolherem e me oferecerem um segundo lar.

Ao orientador da pesquisa, Prof. Dr. Paulo Eigi Miyagi, pela sua constante supervisão e permanente incentivo para o desenvolvimento deste trabalho.

À FAPESP e ao CNPq, que financiaram a realização desta pesquisa.

A todos os colegas da pós-graduação e todos os meus amigos que direta ou indiretamente colaboraram na execução deste trabalho. 


\section{Lista de Abreviaturas ou Siglas}

AE emissão acústica.

AI inteligência artificial

ANN rede neural artificial

ARCOS-SM ARCOS auto-modificáveis

BAS sistema balanceado de produção.

BPN rede de Petri procedural.

CNC comando numérico computadorizado.

CVDS sistemas dinâmicos de variáveis contínuas.

DEDS sistemas dinâmicos a eventos discretos.

DIF detecção e isolamento de falhas.

DPN rede de Petri distribuída.

FMS sistemas flexíveis de manufatura.

GSPN rede de Petri generalizada estocástica.

PN rede de Petri.

PN C/E rede de Petri Condição/Evento.

PN P/T rede de Petri Lugar/Transição

PN-central-E rede de Petri-central-Estendida

SG "Strain Gage"

SMPN rede de Petri Auto-Modificável .

SPSC sistema de processos espontâneos com restrições. 


\section{Lista de Símbolos}

S sub-rede de Petri.

$\mathrm{S}^{*} \quad$ sub-rede de Petri associada.

$\mathrm{Z} \quad$ rede de Petri original.

Z' rede de Petri estendida.

$\mathrm{M}_{0} \quad$ MARCAÇÃo inicial

$\mathrm{M}_{0}^{\prime} \quad$ MARCAÇÃo inicial de Z'.

$\mathrm{M}^{*}{ }_{0}$ MARCAÇÃO inicial de $\mathrm{S}^{*}$.

$\mathrm{R}(\mathrm{PN})$ conjunto de alcançabilidade da rede PN.

$\forall x \quad$ para todo $x$

$\mathrm{X} \in \mathrm{Y} \quad \mathrm{X}$ pertence a $\mathrm{Y}$

$\mathrm{X} \subset \mathrm{Y} \quad \mathrm{X}$ está contido em $\mathrm{Y}$

$\mathbb{N}$ conjunto dos números naturais

$\mathrm{X} \cap \mathrm{Y}$ interseção entre $\mathrm{X}$ e $\mathrm{Y}$

$\varnothing \quad$ conjunto vazio

$\mathrm{P}=\left\{\mathrm{p}_{1}, \mathrm{p}_{2}, \ldots, \mathrm{p}_{\mathrm{m}}\right\}$ : conjunto de LUGARES da rede, $\mathrm{m}=$ número de LUGARES.

$\mathrm{T}=\left\{\mathrm{t}_{1}, \mathrm{t}_{2}, \ldots, \mathrm{t}_{\mathrm{s}}\right\}$ : conjunto de TRANSIÇÕES, $\mathrm{s}=$ número de TRANSIÇÕES.

$T_{n}$ : TRANSIÇÕES normais, definida na PN C/E.

TOR： TRANSIÇÕES “OU”, definida na BPN.

$\mathrm{I}\left(\mathrm{p}_{\mathrm{j}}, \mathrm{t}_{\mathrm{i}}\right)$ : função que define os ARCOS de entrada em relação às TRANSIÇÕES.

$\mathrm{O}\left(\mathrm{t}_{i}, \mathrm{p}_{\mathrm{u}}\right)$ : função que define os ARCOS de saída em relação às TRANSIÇÕES.

$M_{j}=\left(M\left(p_{1}\right), M\left(p_{2}\right), \ldots, M\left(p_{m}\right)\right)$ : MARCAÇÃo no instante $j$.

$u_{\mathrm{k}}$ : $\quad$ vetor $(s x l)$ com grandeza de $\mathbf{1}$ na posição $\boldsymbol{i}$ que representa o disparo da TRANSIÇÃo $t_{i}$ e zero (0) nas outras posições.

$A=O(P, T)-I(P, T)$ : matriz de incidência $(s x m)$.

' $p\left(p^{\bullet}\right)$ : pré-eventos (pós-eventos) do LUGAR p.

•t $\left(t^{\bullet}\right)$ : pré-condições (pós-condições) da TRANSIÇão t. 